\title{
Identification of the unknown coefficient in a quasi-linear parabolic equation by a semigroup approach
}

Ebru Ozbilge ${ }^{* *}$ and Ali Demir ${ }^{2}$

\section{*Correspondence:}

ebru.ozbilge@ieu.edu.tr

'Department of Mathematics,

Faculty of Science and Literature,

Izmir University of Economics,

Sakarya Caddesi, No. 156, Balcova,

Izmir 35330, Turkey

Full list of author information is

available at the end of the article

\begin{abstract}
This article presents a semigroup approach to the mathematical analysis of the inverse coefficient problems of identifying the unknown coefficient $a(x, t)$ in the quasi-linear parabolic equation $u_{t}(x, t)=u_{x x}(x, t)+a(x, t) u(x, t)$ with Dirichlet boundary conditions $u(0, t)=\psi_{0}, u(1, t)=\psi_{1}$. It is shown that the unknown coefficient $a(x, t)$ can be approximately determined via the semigroup approach.
\end{abstract}

\section{Introduction}

Consider the following initial boundary value problem:

$$
\left\{\begin{array}{l}
u_{t}(x, t)=u_{x x}(x, t)+a(x, t) u(x, t), \quad(x, t) \in \Omega_{T}, \\
u(x, 0)=g(x), \quad 0<x<1, \\
u(0, t)=\psi_{0}, \quad u(1, t)=\psi_{1}, \quad 0<t<T,
\end{array}\right.
$$

where $\Omega_{T}=\left\{(x, t) \in R^{2}: 0<x<1,0<t \leq T\right\}$. The left and right boundary values $\psi_{0}, \psi_{1}$ are assumed to be constants. The functions $c_{1}>a(x, t) \geq c_{0}>0$ and $g(x)$ satisfy the following conditions:

(C1) $a(x, t) \in H^{1,2}\left(\Omega_{T}\right)$;

(C2) $g(x) \in H^{3,2}[0,1], g(0)=\psi_{0}, g(1)=\psi_{1}$.

Under these conditions, the initial boundary value problem (1) has the unique solution $u(x, t) \in H^{2,2}[0,1] \cap H^{1,2}[0,1][1-3]$.

Consider the inverse problem of determining the unknown coefficient $a(x, t)$ from the following observations at the boundary $x=0$ :

$$
u_{x}(0, t)=f(t), \quad t \in(0, T]
$$

Here $u=u(x, t)$ represents the solution of the parabolic problem (1). It is assumed that the function $f(t)$ is noisy free measured output data. In this context the parabolic problem (1) will be referred to as a direct (forward) problem with the inputs $g(x)$ and $a(x, t)$. The function $f(t)$ is assumed to belong to $H^{1,2}[0, T]$ and satisfy the consistency conditions $f(0)=g^{\prime}(0)$. Moreover, it is assumed that the function $g(x)$ satisfies the consistency conditions $g(0)=\psi_{0}, g(1)=\psi_{1}$.

\section{Springer}

(O) 2013 Ozbilge and Demir; licensee Springer. This is an Open Access article distributed under the terms of the Creative Commons Attribution License (http://creativecommons.org/licenses/by/2.0), which permits unrestricted use, distribution, and reproduction in any medium, provided the original work is properly cited. 
In inverse problems, mostly numerical methods are employed to determine the unknown function. Even using one or more measured output data in analytic methods does not help in determination of the unknown function exactly $[4,5]$.

The purpose of this paper is to determine the analytic representation of the approximate unknown coefficient $a(x, t)$ via a semigroup approach. We determine the approximate solution of the boundary value problem (1) without determining the unknown coefficient $a(x, t)$. In the determination of it, we just use the values $a(0,0), a_{x}(0,0)$ which are obtained and the over measured data $u_{x}(0, t)=f(t)$. However, this cannot be possible for any kind of a boundary value problem with different type of boundary conditions. The semigroup approach for inverse problems for the identification of unknown coefficient in a quasi-linear parabolic equations was studied by Demir and Ozbilge [6, 7]. Moreover, the identification of the unknown diffusion coefficient in a linear parabolic equation was studied by Demir and Hasanov [8]. The study in this paper is based on similar philosophy to that in [6-9].

Recent studies [6-9] on the identification of the unknown function via a semigroup approach [10] show that the semigroup approach is the most effective analytical method which sheds more light on the unknown function. The weak point of this method is that you cannot apply this method to the initial boundary value problems with boundary conditions other than constant or zero. Overcoming this problem in some way makes this method more effective and valuable.

The paper is organized as follows. In Section 2, determination of the unknown coefficient $a(x, t)$ is given via the semigroup approach. Some concluding remarks are given in Section 3.

\section{Determination of the unknown coefficient $a(x, t)$ via the semigroup approach}

Consider the inverse problem of determining the explicit form of the unknown coefficient $a(x, t)$ via the semigroup approach. Let the parabolic equation be arranged as follows:

$$
u_{t}(x, t)-u_{x x}(x, t)=a(x, t) u(x, t), \quad(x, t) \in \Omega_{T} .
$$

Here we treat the term $a(x, t) u(x, t)$ as a right-hand side function, i.e., $F(x, t)=a(x, t) u(x, t)$. In order to formulate the solution of the parabolic problem (1) in terms of a semigroup, a new function needs to be defined to make the boundary values equal to zero:

$$
v(x, t)=u(x, t)-\psi_{0}(1-x)-\psi_{1} x, \quad x \in[0,1]
$$

which satisfies the following parabolic problem:

$$
\begin{aligned}
& v_{t}(x, t)+A[v(x, t)]=a(x, t)\left(v(x, t)+\psi_{0}(1-x)+\psi_{1} x\right), \quad(x, t) \in \Omega_{T}, \\
& v(x, 0)=g(x)-\psi_{0}(1-x)-\psi_{1} x, \quad 0<x<1, \\
& v(0, t)=0, \quad v(1, t)=0, \quad 0<t<T .
\end{aligned}
$$

We can represent the solution of this problem in terms of a semigroup of linear operators. Here, $A[\cdot]:=-\frac{d^{2}[\cdot]}{d x^{2}}$ is a second-order differential operator and its domain is $D_{A}=\{v(x) \in$ $\left.H_{0}^{2,2}(0,1) \cap H_{0}^{3,2}[0,1]: v(0)=0=v(1)\right\}$, where $H_{0}^{2,2}(0,1)=\overline{C_{0}^{2}(0,1)}$ and $H_{0}^{1,2}[0,1]=\overline{C_{0}^{1}[0,1]}$ 
are Sobolev spaces. Obviously, by completion $g(x) \in D_{A}$ since the initial value function $g(x)$ belongs to $C^{3}[0,1]$. Hence $D_{A}$ is dense in $H_{0}^{2,2}[0,1]$, which is a necessary condition for being an infinitesimal generator.

In the following, despite doing the calculations in the smooth function space, by completion they are valid in the Sobolev space. Denote by $T(t)$ the semigroup of linear operators generated by the operator $-A[5,6]$. Since the initial boundary value problem (4) possesses the property of uniqueness of its solutions, then the solution $v_{h}(x, t)$ of its homogeneous part can be written as follows:

$$
v_{h}(x, t)=T(t) v(x, 0)=T(t)\left(g(x)-\psi_{0}(1-x)-\psi_{1} x\right) .
$$

Furthermore, by using the variations of parameters formula, we can write the solution of the initial boundary value problem (3) as follows:

$$
v(x, t)=T(t) v(x, 0)+\int_{0}^{t} T(t-s)\left(a(x, s)\left(v(x, s)+\psi_{0}(1-x)+\psi_{1} x\right)\right) d s .
$$

In order to construct the semigroup $T(t)$, we need to identify the eigenvalues and eigenfunctions of the infinitesimal operator $A$. Hence, the following eigenvalue problem must first be considered:

$$
\begin{aligned}
& A \phi(x)=\lambda \phi(x), \\
& \phi(0)=0 ; \quad \phi(1)=0 .
\end{aligned}
$$

This problem is called the Sturm-Liouville problem. The eigenvalues are determined with $\lambda_{n}=n^{2} \pi^{2}$ for all $n=0,1, \ldots$ the corresponding eigenfunctions as $\phi_{n}(x)=\sqrt{2} \sin (n \pi x)$. In this case, the semigroup $T(t)$ can be represented in the following way:

$$
T(t) U(x, s)=\sum_{n=0}^{\infty}\left\langle\phi_{n}(\theta), U(\theta, s)\right\rangle e^{-\lambda_{n} t} \phi_{n}(x),
$$

where $\left\langle\phi_{n}(\theta), U(\theta, s)\right\rangle=\int_{0}^{1} \phi_{n}(\theta) U(\theta, s) d \theta$. It is well known that the Sturm-Liouville problem (5) generates a complete orthogonal family of eigenfunctions so that the null space of the semigroup $T(t)$ is trivial, i.e., $N(T)=\{0\}$. The null space of the semigroup $T(t)$ of the linear operators can be defined as follows:

$$
N(T)=\left\{U(\theta, t):\left\langle\phi_{n}(\theta), U(\theta, t)\right\rangle=0, \text { for all } n=0,1,2,3, \ldots\right\}
$$

The unique solution of the initial-boundary value problem (4) in terms of the semigroup $T(t)$ can be represented in the following form:

$$
v(x, t)=T(t) v(x, 0)+\int_{0}^{t} T(t-s)\left(a(x, s)\left(v(x, s)+\psi_{0}(1-x)+\psi_{1} x\right)\right) d s .
$$

Hence, by using identity (3) and taking the initial value $u(x, 0)=g(x)$ into account, the solution $u(x, t)$ of the parabolic problem (1) in terms of a semigroup can be written in the 
following form:

$$
\begin{aligned}
u(x, t)= & \psi_{0}(1-x)+\psi_{1} x+T(t)\left(g(x)-\psi_{0}(1-x)-\psi_{1} x\right) \\
& +\int_{0}^{t} T(t-s) a(x, s) u(x, s) d s
\end{aligned}
$$

Now, differentiating both sides of identity (7) with respect to $t$ yields

$$
\begin{aligned}
u_{t}(x, t)= & -T(t) A\left(u(x, 0)-\psi_{0}(1-x)-\psi_{1} x\right)+a(x, t) u(x, t) \\
& -\int_{0}^{t} A T(t-s) a(x, s) u(x, s) d s .
\end{aligned}
$$

Using the semigroup property $-\int_{0}^{t} A T(t-s) u(x, s) d s=T(0) u(x, t)-T(t) u(x, 0)$,

$$
u_{t}(x, t)=-T(t) g^{\prime \prime}(x)+2 a(x, t) u(x, t)-T(t) a(x, 0) u(x, 0)
$$

is obtained. Taking $t=0$ in the above identity, we get

$$
u_{t}(x, 0)=-g^{\prime \prime}(x)+a(x, 0) u(x, 0)
$$

Taking $x=0$ in the above identity, we obtain

$$
u_{t}(0,0)=-g^{\prime \prime}(0)+a(0,0) u(0,0) .
$$

Since $u(0, t)=\psi_{0}, u_{t}(0, t)=0$. Taking this into account yields

$$
0=-g^{\prime \prime}(0)+a(0,0) g(0)
$$

By solving the equation for $a(0,0)$, the following explicit formula for the value $a(0,0)$ of the unknown coefficient $a(x, t)$ is obtained:

$$
a(0,0)=\frac{g^{\prime \prime}(0)}{g(0)}
$$

Now, differentiating both sides of identity (8) with respect to $x$ yields

$$
u_{x t}(x, 0)=-g^{\prime \prime \prime}(x)+a_{x}(x, 0) u(x, 0)+a(x, 0) u_{x}(x, 0) .
$$

Taking $x=0$ in the above identity, we obtain

$$
u_{x t}(0,0)=-g^{\prime \prime \prime}(0)+a_{x}(0,0) u(0,0)+a(0,0) u_{x}(0,0) .
$$

Since $u_{x}(0, t)=f(t), u_{x t}(0, t)=f^{\prime}(t)$. Taking this into account yields

$$
f^{\prime}(0)=-g^{\prime \prime \prime}(0)+a_{x}(0,0) g(0)+\frac{g^{\prime \prime}(0)}{g(0)} f(0)
$$


By solving the equation for $a_{x}(0,0)$, the following explicit formula for the value $a_{x}(0,0)$ of the unknown coefficient $a(x, t)$ is obtained:

$$
a_{x}(0,0)=\frac{g^{\prime \prime \prime}(0) g(0)-g^{\prime \prime}(0) f(0)+f^{\prime}(0) g(0)}{g^{2}(0)} .
$$

By making use of the values $a(0,0)$ and $a_{x}(0,0)$, we can write a linear approximation for $a(x, 0)$ near $(0,0)$ as follows:

$$
a(x, 0)=\frac{g^{\prime \prime}(0)}{g(0)}+\frac{g^{\prime \prime \prime}(0) g(0)-g^{\prime \prime}(0) f(0)+f^{\prime}(0) g(0)}{g^{2}(0)} x .
$$

Substituting the linear approximation (11) into equation (8) yields

$$
u_{t}(x, 0)=-g^{\prime \prime}(x)+\left(\frac{g^{\prime \prime}(0)}{g(0)}+\frac{g^{\prime \prime \prime}(0) g(0)-g^{\prime \prime}(0) f(0)+f^{\prime}(0) g(0)}{g^{2}(0)} x\right) u(x, 0),
$$

which enables us to obtain the approximate solution of the parabolic problem (1). For this, we first need to show that

$$
T(s) u_{t}(x, t)=u_{t}(x, s+t)
$$

by using the well-known property of the semigroup of the linear operators

$$
T(s) u(x, t)=u(x, s+t)
$$

which leads us to the determination of the unknown coefficient $a=a(x, t)$ analytically.

Lemma Let $T(t)$ be the semigroup of the linear operators, and let $u(x, t)$ be the solution of the equation $u_{t}(x, t)=A u(x, t)$, where $A$ is the infinitesimal generator of $T(t)$. Then the following identity holds:

$$
T(s) u_{t}(x, t)=u_{t}(x, s+t)
$$

for each $t, s \in(0, T]$.

Proof First of all, observe that since eigenfunctions $\phi_{n}(x)$ of the infinitesimal generator $A$ generate a complete orthogonal family, we can write $u(x, t)$ in terms of eigenfunctions in the following form:

$$
u(x, t)=\sum_{n=0}^{\infty}\left\langle\phi_{n}(\cdot), u(\cdot, t)\right| \phi_{n}(x)
$$

Taking derivative of both sides with respect to the variable $t$ produces

$$
u_{t}(x, t)=\sum_{n=0}^{\infty} \frac{d}{d t}\left\langle\phi_{n}(\cdot), u(\cdot, t)\right| \phi_{n}(x)
$$


Notice that this identity implies that $u_{t}(x, t)$ is in the domain of the semigroup of linear operators $T(t)$. Hence we can apply $T(t)$ to $u_{t}(x, t)$ :

$$
T(s) u_{t}(x, t)=\sum_{n=0}^{\infty} \frac{d}{d t}\left\langle\phi_{n}(\cdot), u(\cdot, t)\right| e^{-\lambda_{n} s} \phi_{n}(x) .
$$

Now we know that

$$
u(x, s+t)=T(s) u(x, t)=\sum_{n=0}^{\infty}\left\langle\phi_{n}(\cdot), u(\cdot, t)\right| e^{-\lambda_{n} s} \phi_{n}(x) .
$$

Taking derivative of both sides with respect to the variable $t$ produces

$$
u_{t}(x, s+t)=\sum_{n=0}^{\infty} \frac{d}{d t}\left\langle\phi_{n}(\cdot), u(\cdot, t)\right\rangle e^{-\lambda_{n} s} \phi_{n}(x)
$$

which is exactly the same as (11). This is exactly what is desired. However, notice that the identity

$$
T(s) u_{x}(x, t)=u_{x}(x, s+t)
$$

does not hold.

This lemma leads us to the solution of the parabolic problem (1) without knowing the unknown coefficient $a(x, t)$, but knowing $a(0,0)$ and $a_{x}(0,0)$. Then the solution enables us to determine the unknown coefficient $a(x, t)$ approximately.

Now, applying the identity (13) to (12) and using the linearity, we get

$$
\begin{aligned}
u_{t}(x, t) & =T(t) u_{t}(x, 0) \\
& =-T(t) g^{\prime \prime}(x)+\left(\frac{g^{\prime \prime}(0)}{g(0)}+\frac{g^{\prime \prime \prime}(0) g(0)-g^{\prime \prime}(0) f(0)+f^{\prime}(0) g(0)}{g^{2}(0)} x\right) T(t) g(x) .
\end{aligned}
$$

Hence we can obtain the solution $u(x, t)$ from the above identity by integrating with respect to $t$ as follows:

$$
\begin{aligned}
u(x, t)= & \int\left(-T(t) g^{\prime \prime}(x)\right. \\
& \left.+\left(\frac{g^{\prime \prime}(0)}{g(0)}+\frac{g^{\prime \prime \prime}(0) g(0)-g^{\prime \prime}(0) f(0)+f^{\prime}(0) g(0)}{g^{2}(0)} x\right) T(t) g(x)\right) d t+C(x),
\end{aligned}
$$

where $C(x)$ denotes the integration constant. We can determine it by using the initial condition

$$
\begin{aligned}
u(x, 0)= & \left(\int \left(-T(t) g^{\prime \prime}(x)\right.\right. \\
& \left.\left.+\left(\frac{g^{\prime \prime}(0)}{g(0)}+\frac{g^{\prime \prime \prime}(0) g(0)-g^{\prime \prime}(0) f(0)+f^{\prime}(0) g(0)}{g^{2}(0)} x\right) T(t) g(x)\right) d t\right)_{t=0}+C(x),
\end{aligned}
$$


which yields

$$
\begin{aligned}
C(x)= & g(x)-\left(\int \left(-T(t) g^{\prime \prime}(x)\right.\right. \\
& \left.\left.+\left(\frac{g^{\prime \prime}(0)}{g(0)}+\frac{g^{\prime \prime \prime}(0) g(0)-g^{\prime \prime}(0) f(0)+f^{\prime}(0) g(0)}{g^{2}(0)} x\right) T(t) g(x)\right) d t\right)_{t=0} .
\end{aligned}
$$

Substituting the integration constant $C(x)$ into the identity (15), we obtain the approximate solution of the parabolic problem (1). Hence, by using this solution and the parabolic equation, we are able to determine the unknown coefficient $a(x, t)$ approximately.

\section{Conclusion}

The aim of this study was to determine the unknown coefficient $a(x, t)$ via the semigroup approach. Lemma in Section 2 plays a crucial role in the identification of the unknown coefficient $a(x, t)$. This lemma leads us to new conclusions about the identification problems $v i a$ the semigroup approach. We will present these results in our future studies....

\section{Competing interests}

The authors declare that they have no competing interests.

\section{Authors' contributions}

Both authors contributed equally to the manuscript and read and approved the final manuscript.

\section{Author details}

${ }^{1}$ Department of Mathematics, Faculty of Science and Literature, Izmir University of Economics, Sakarya Caddesi, No. 156, Balcova, Izmir 35330, Turkey. ${ }^{2}$ Department of Mathematics, Kocaeli University, Umuttepe, Izmit, Kocaeli 41380, Turkey.

\section{Acknowledgements}

Dedicated to Professor Hari M Srivastava.

The research was supported by parts by the Scientific and Technical Research Council (TUBITAK) of Turkey and Izmir University of Economics.

Received: 26 November 2012 Accepted: 13 April 2013 Published: 26 April 2013

\section{References}

1. Showalter, R: Monotone Operators in Banach Spaces and Nonlinear Partial Differential Equations. Am. Math. Soc. Providence (1997)

2. Hasanov, A, Demir, A, Erdem, A: Monotonicity of input-output mappings in inverse coefficient and source problem for parabolic equations. J. Math. Anal. Appl. 335, 1434-1451 (2007)

3. Hasanov, A, DuChateau, P, Pektas, B: An adjoint approach and coarse-fine mesh method for identification of the diffusion coefficient in a linear parabolic equations. J. Inverse III-Posed Probl. 14, 435-463 (2006)

4. DuChateau, P, Gottlieb, J: Introduction to Inverse Problems in Partial Differential Equations for Engineers, Physicists and Mathematicians. Kluwer Academic, Amsterdam (1996)

5. Renardy, M, Rogers, R: An Introduction to Partial Differential Equations. Springer, New York (2004)

6. Demir, A, Ozbilge, E: Analysis of a semigroup approach in the inverse problem of identifying an unknown coefficient. Math. Methods Appl. Sci. 31, 1635-1645 (2008)

7. Demir, A, Ozbilge, E: Semigroup approach for identification of the unknown diffusion coefficient in a quasi-linear parabolic equation. Math. Methods Appl. Sci. 30, 1283-1294 (2007)

8. Demir, A, Hasanov, A: Identification of the unknown diffusion coefficient in a linear parabolic equation by the semigroup approach. J. Math. Anal. Appl. 340, 5-15 (2008)

9. Ozbilge, E: Identification of the unknown diffusion coefficient in a quasi-linear parabolic equation by semigroup approach with mixed boundary conditions. Math. Methods Appl. Sci. 31, 1333-1344 (2008)

10. Ashyralyev, A, San, ME: An approximation of semigroup method for stochastic parabolic equations. Abstr. Appl. Anal. (in press) 